\title{
A female patient with GSD IXc developing multiple and recurrent hepatocellular carcinoma: a case report and literature review
}

Jun Kido ${ }^{1,2 \bowtie}$, Hiroshi Mitsubuchi ${ }^{1,3}$, Takehisa Watanabe ${ }^{4}$, Keishin Sugawara ${ }^{2}$, Hideo Sasai ${ }^{5}$, Toshiyuki Fukao ${ }^{5}$ and Kimitoshi Nakamura ${ }^{1,2}$

(C) The Author(s) 2021

Glycogen storage disease type IX (GSD IX), the most common form of GSD, is caused by a defect in phosphorylase kinase (PhK). We describe the case of a female patient with GSD IXc harboring a homozygous mutation in PHKG2 (NM_000294.3; PHKG2 (c.280_282delATC (p. 194del)) definitively diagnosed using the GSD gene panel. She presented with hypoglycemia, hepatomegaly, and short stature and died of cirrhosis and recurrent multiple hepatocellular adenoma at the age of 69 years and 11 months.

Human Genome Variation (2021) 8:1-6; https://doi.org/10.1038/s41439-021-00172-8

Glycogen storage disease type IX (GSD IX) is one of the most common forms of GSD caused by a defect in phosphorylase kinase (PhK). This disease accounts for $25 \%$ of all GSD cases, with an estimated frequency of 1 in 100,000 individuals ${ }^{1}$. PhK (EC 2.7.1.38) is a serine/threonine-specific complex protein kinase consisting of four subunits: alpha $(a)$, beta $(\beta)$, gamma $(\gamma)$, and delta $(\delta)^{2}$. PhK activates glycogen phosphorylase (EC 2.4.1.1), which releases glucose-1-phosphate from glycogen ${ }^{3}$. Patients with GSD IX have elevated transaminases and hepatomegaly, growth retardation, hypertriglyceridemia, and hypercholesterolemia. However, compared to that in patients with other types of liver GSDs, clinical manifestation in GSD IX patients can be mild, and patients may become asymptomatic as they age $\mathrm{e}^{4,5}$. Mutations in PHKA1, PHKA2, or PHKB and PHKG2 cause GSD IX. PHKG2 encodes the hepatic isoform of the $\gamma$ unit of PhK. Mutation in PHKG2 causes GSD IXc (MIM: 613027), a rare form that manifests as hepatomegaly, hypotonia, and growth retardation in childhood. Although these symptoms improve with age, some patients develop hepatic fibrosis, cirrhosis, and liver dysfunction ${ }^{6}$.

A female patient was definitively diagnosed with GSD IXc at the age of 68 years using the GSD gene panel. She was diagnosed with hepatic GSD in childhood but self-discontinued clinical examination and treatment; later, she developed liver cirrhosis and hepatocellular carcinoma (HCC). Herein we present her clinical course and discuss the mechanism and treatment of HCC in GSD IXc based on her clinical course.

A 67-year-old female patient with suspected GSD was introduced to our institution to receive a definitive diagnosis while receiving conservative care therapy for liver cirrhosis and multiple HCC. She was the first child of healthy consanguineous parents. Her height was $141.9 \mathrm{~cm}$ (the average Japanese female height at $65-69$ years is $153.3 \pm 5.7 \mathrm{~cm}$ ), and her weight was
$54.7 \mathrm{~kg}$. She presented with anasarca and ascites due to cirrhosis and HCC. She had no drinking habit and no history of hepatitis B or $\mathrm{C}$ infection. She also had no history of blood transfusion before receiving hepatectomy at the age of 56 years. Her intelligence was not impaired, and she could write advanced sentences. Hepatomegaly was first detected in this patient at 7 years; however, the diagnosis was not completed. At 12 years, she was diagnosed with GSD (type: unknown) at our institution, but she self-discontinued regular follow-up. At 30 years of age, she visited the Department of Internal Medicine because of frequent upper abdominal pain and fasting hypoglycemia symptoms, such as irritation and wooziness. In addition, she developed multiple adenomas and cirrhosis of the liver and received regular follow-up and medicine, including a gastrointestinal drug every month (Supplemental Data 1). She gradually lost the ability to work due to fatigue and hypoglycemia symptoms and lived a single life, receiving a pension under the social security system. At the age of 56 years, she underwent S7 partial hepatectomy because of HCC (Fig. 1A-E). At 63 years, she experienced multiple HCC relapse and underwent combination therapy of S4 partial hepatectomy and radiofrequency ablation (Fig. 1F). At the age of 66 years, she developed esophageal varix and underwent endoscopic variceal ligation $(E V L)$, and she developed multiple recurrent HCCs and underwent transcatheter arterial chemoembolization (TACE) at the age of 67 years. In addition, she developed hepatic coma after rupture of the esophageal varix, which was rescued by EVL. At the age of 68 years, she developed recurrent multiple HCC and underwent a second TACE. After that, she could not undergo aggressive therapy for recurrent HCC because of impaired hepatic function and liver cirrhosis (Fig. 1G and Supplemental Data 1).

She was definitively diagnosed with GSD IXc because she harbored a homozygous c.280_282delATC (p. Ile94del) mutation

\footnotetext{
${ }^{1}$ Department of Pediatrics, Kumamoto University Hospital, Kumamoto, Japan. ${ }^{2}$ Department of Pediatrics, Faculty of Life Sciences, Kumamoto University, Kumamoto, Japan. ${ }^{3}$ Department of Neonatology, Kumamoto University Hospital, Kumamoto, Japan. ${ }^{4}$ Department of Gastroenterology and Hepatology, Faculty of Life Sciences, Kumamoto University, Kumamoto, Japan. ${ }^{5}$ Department of Pediatrics, Graduate School of Medicine, Gifu University, Gifu, Japan. ${ }^{凶}$ email: kidojun@kuh.kumamoto-u.ac.jp
}

Received: 7 August 2021 Revised: 6 October 2021 Accepted: 9 October 2021

Published online: 8 December 2021 

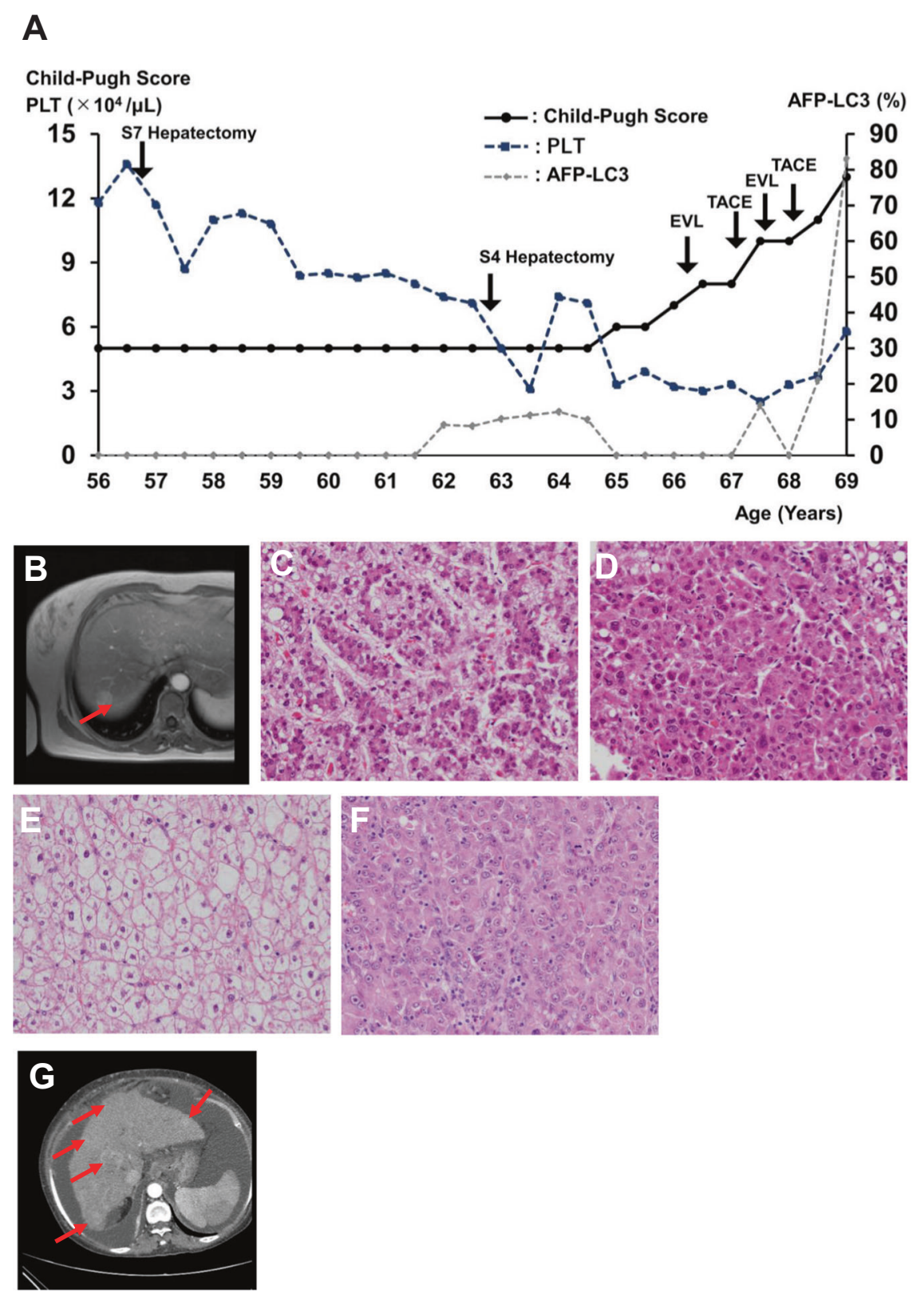

Fig. 1 Hepatocellular carcinoma (HCC) in a female patient with GSD IXc. A Clinical course. Deterioration of cirrhosis and HCC progressed with age. B Liver MRI. T2-weighted imaging detected a 2-cm tumor in the liver (S7 section). C-F Pathological tissues. The S7 tumor exhibited characteristics of well-differentiated (C) and moderately differentiated (D) HCC combined with hepatocytes with a clear cell cytoplasm (E), consistent with pathology in GSD. The S4 tumor was poorly differentiated (F) and moderately differentiated HCC. G Liver CT. Multiple HCC and ascites were detected in the abdomen.

in PHKG2. She died under palliative care at 69 years and 11 months.

Written informed consent was obtained from the patient's parents. This study was approved by the Institutional Ethics Committee of the Faculty of Life Science, Kumamoto University.

We present a female patient with GSD IXc who developed liver cirrhosis and multiple HCC. She underwent aggressive surgical and medical treatment until the age of 68 years. However, she did not modify her diet, including consuming uncooked cornstarch for GSD, owing to her specific characteristics, including being stubborn, obsessive, and unwilling to accept others' opinions. She was not aware of the need to follow a diet suitable for preventing hypoglycemia. To our knowledge, this is the first report of a patient with GSD IXc developing multiple HCC and receiving aggressive surgical and medical treatment. She carried a novel mutation in PHKG2. We have summarized the case report of GSD IXc in Table 1 (Supplemental Data 2).

The clinical outcome in 30 patients with GSD IXc was collected in an earlier study ${ }^{7}$. Hepatomegaly, fasting hypoglycemia, fasting ketosis, hypertriglyceridemia, hypercholesterolemia, growth delay, developmental delay, and elevated transaminase were present in $100 \%(30 / 30), 94.7 \%(18 / 19)$, $100 \%$ (6/6), $94.4 \%$ (17/18), 45.4\% (5/11), 70.8\% (17/24), 50\% (10/ $20)$, and $100 \%(22 / 22)$ of patients with GSD IXc, respectively. Eighty percent $(24 / 30)$ of patients underwent a liver biopsy. According to pathology reports, $95.8 \%$ of patients $(23 / 24)$ presented with either fibrosis (mild, moderate, or severe) or cirrhosis. Three patients developed a hepatic adenoma. Only one patient developed HCC at the age of 27 years; this patient is awaiting liver transplantation. 


:

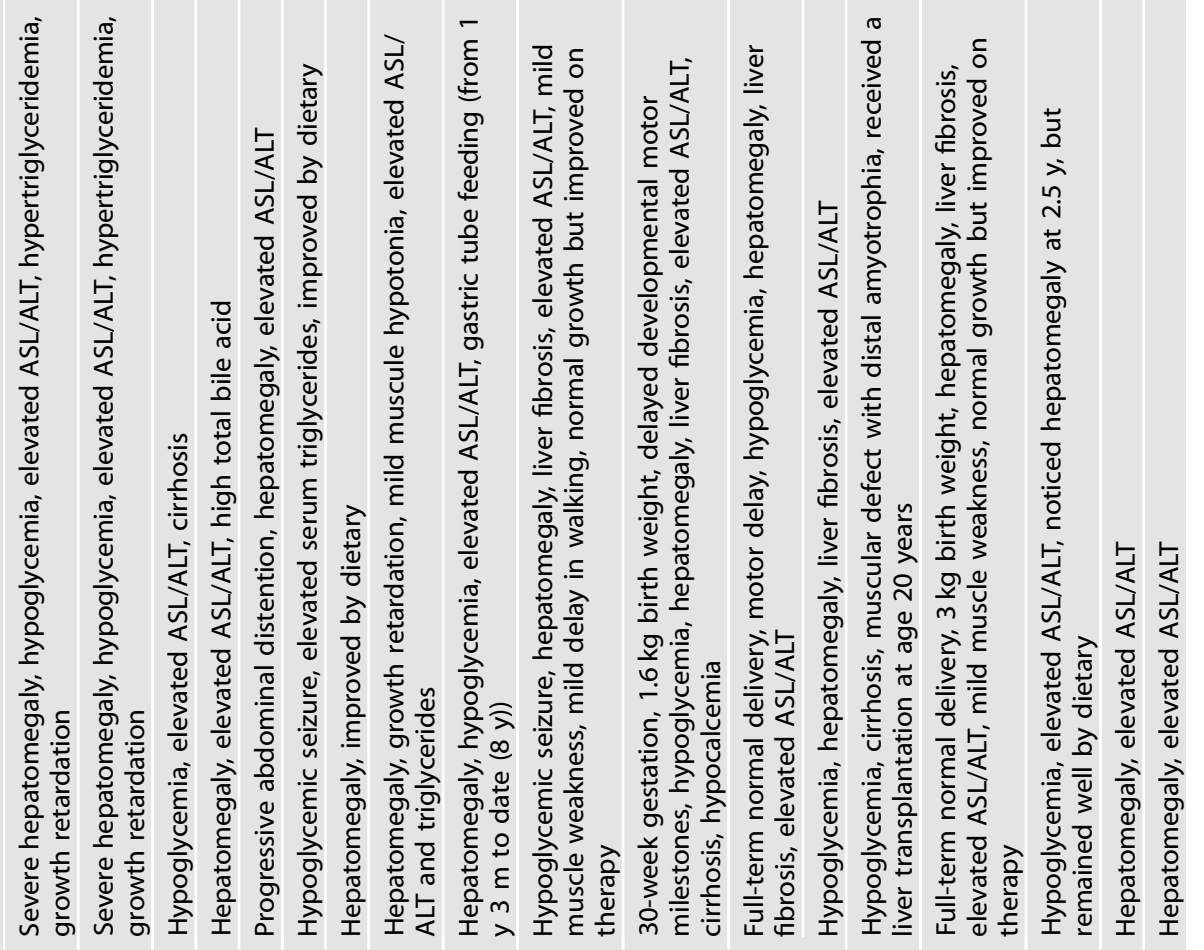

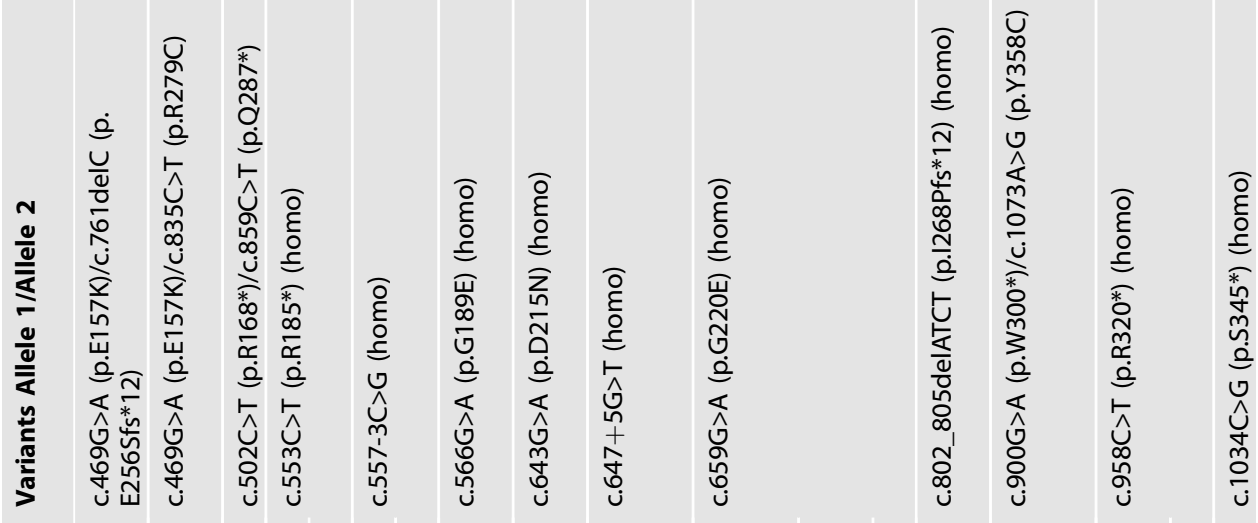

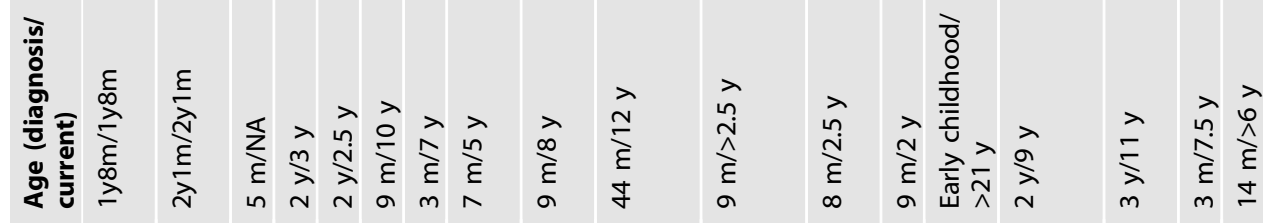

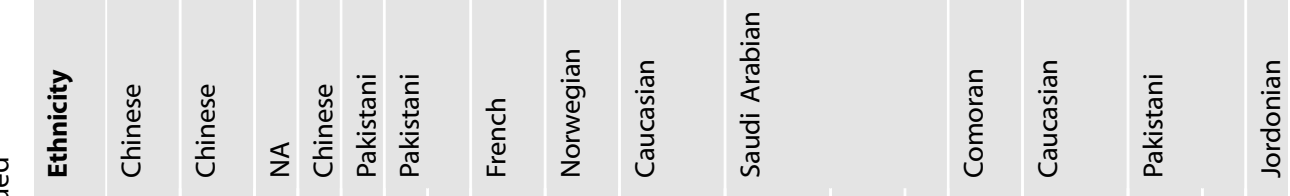

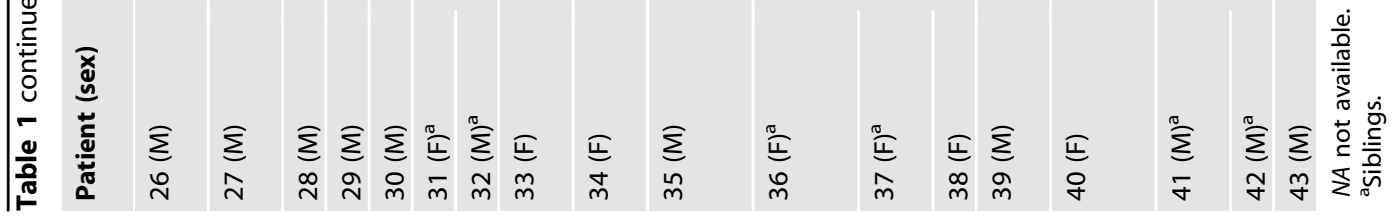


There is no consensus on effective treatment for GSD IXc. Nasogastric tube feeding, uncooked cornstarch $(0.3-2.0 \mathrm{~g} / \mathrm{kg}$ body weight (BW)/day), and a high-protein diet (3.0-4.0 g/kg BW/day) are common treatments for GSD IX $\mathrm{Xc}^{4,8,9}$. Poor metabolic control is a risk factor for the development of long-term complications such as hepatocellular adenoma in hepatic GSD ${ }^{10-13}$. Moreover, hepatocellular adenoma may be regressed in a well-controlled metabolic state with strict dietary therapy. Nevertheless, the effectiveness of strict diet therapy is controversial because there are few adult patients with GSD IXc who receive long-term strict diet therapy.

We believe that this patient should have received a liver transplant when she was diagnosed with HCC. We had reservations in treating her HCC, which developed at the age of 58 years, particularly with regard to choosing between aggressive invasive treatment, including hepatectomy and TACE, and conservative treatment because HCC is known to recur. Overall, it is important to control the radical metabolic state in metabolic disease.

Cirrhosis is the most important risk factor for the development of HCC, regardless of etiology ${ }^{14}$. Liver fibrosis and hepatocellular carcinoma occur in patients with GSD VI ${ }^{15,16}$ caused by a defect in glycogen phosphorylase. GSD IXc with pathogenic variants in PHKG2 is associated with more severe clinical and biochemical abnormalities, including increased risk for liver fibrosis and cirrhosis $^{15,17,18}$, because $\gamma$ is the catalytic subunit, whereas a and $\beta$ are regulatory subunits of PhK. Glycogen is a key energy store for cancer cells ${ }^{19}$, and glycogen turnover allows cancer cells to adapt and survive under adverse oxygen and nutrient conditions within the tumor microenvironment. Glycogen breakdown supports the pentose phosphate pathway, which generates the nucleotides required for proliferation and DNA repair, and NADPH, which is an important reducing agent for reactive oxygen species scavenging and for the synthesis of nucleotides, amino acids, and lipids ${ }^{19}$. Therefore, both cirrhosis and glycogen accumulation are important factors in recurrent and multiple HCC. Activated PhK $\beta$-subunit expression regulates the occurrence of HCC by inhibiting AKT/protein kinase $B$ and signal transducer and activator of transcription 3 signaling pathway activation, independent of the glycogenolytic pathway ${ }^{20}$. In hepatic GSD, the formation and proliferation of HCC may be related to a mechanism independent of the glycogenolytic pathway, which should be investigated in the future.

The specific characteristics of our patient might be a clinical manifestation of GSD IXc. We should have realized this possibility earlier and administered appropriate counseling.

In conclusion, we present the case of a patient with GSD IXc who developed liver cirrhosis and multiple HCC. This case shows the long-term clinical course in GSD IXc. Liver cirrhosis and HCC were important complications in this long-term surviving patient with GSD IXc. However, there is no established effective therapy for GSD IXC, and it is not clear to what extent HCC should be aggressively treated with invasive treatment. Knowledge of clinical outcomes in more patients with GSD IXc is essential to design a treatment course for GSD IXc.

\section{HGV DATABASE}

The relevant data from this Data Report are hosted at the Human Genome Variation Database at https://doi.org/10.6084/m9.figshare.hgv.3103.

\section{REFERENCES}

1. Maichele, A. J., Burwinkel, B., Maire, I., Søvik, O. \& Kilimann, M. W. Mutations in the testis/liver isoform of the phosphorylase kinase $\gamma$ subunit (PHKG2) cause autosomal liver glycogenosis in the gsd rat and in humans. Nat. Genet. 14, 337-340 (1996).

2. Brushia, R. J. Phosphorylase kinase: the complexity of its regulation is reflected in the complexity of its structure. Front. Biosci. 4, d618 (1999).

3. Newgard, C. B., Hwang, P. K. \& Fletterick, R. J. The family of glycogen phosphorylases: structure and function. Crit. Rev. Biochem. Mol. Biol. 24, 69-99 (1989).
4. Burwinkel, B., Tanner, M. S. \& Kilimann, M. W. Phosphorylase kinase deficient liver glycogenosis: progression to cirrhosis in infancy associated with PHKG2 mutations (H144Y and L225R). J. Med. Genet. 37, 376-7 (2000).

5. Morava, E. et al. Biochemical characteristics and increased tetraglucoside excretion in patients with phosphorylase kinase deficiency. J. Inherit. Metab. Dis. 28, 703-706 (2005).

6. Burwinkel, B., Shiomi, S., Al Zaben, A. \& Kilimann, M. W. Liver glycogenosis due to phosphorylase kinase deficiency: PHKG2 gene structure and mutations associated with cirrhosis. Hum. Mol. Genet. 7, 149-154 (1998).

7. Fernandes, S. A., Cooper, G. E., Gibson, R. A. \& Kishnani, P. S. Benign or not benign? Deep phenotyping of liver glycogen storage disease IX. Mol. Genet. Metab. 131, 299-305 (2020).

8. Burwinkel, B., Rootwelt, T., Kvittingen, E. A., Chakraborty, P. K. \& Kilimann, M. W. Severe phenotype of phosphorylase kinase-deficient liver glycogenosis with mutations in the PHKG2 gene. Pediatr. Res. 54, 834-839 (2003).

9. Davit-Spraul, A. et al. Liver glycogen storage diseases due to phosphorylase system deficiencies: diagnosis thanks to non invasive blood enzymatic and molecular studies. Mol. Genet. Metab. 104, 137-143 (2011).

10. Minarich, L. A., Kirpich, A., Fiske, L. M. \& Weinstein, D. A. Bone mineral density in glycogen storage disease type la and lb. Genet. Med. 14, 737-741 (2012).

11. Melis, D. et al. Impaired bone metabolism in glycogen storage disease type 1 is associated with poor metabolic control in type 1a and with granulocyte colonystimulating factor therapy in type 1b. Horm. Res. Paediatr. 81, 55-62 (2014).

12. Beegle, R. D., Brown, L. M. \& Weinstein, D. A. Regression of hepatocellular adenomas with strict dietary therapy in patients with glycogen storage disease type I. JIMD Rep. 18, 23-32 (2014).

13. Burda, P. \& Hochuli, M. Hepatic glycogen storage disorders. Curr. Opin. Clin. Nutr. Metab. Care 18, 415-421 (2015).

14. Balogh, J. et al. Hepatocellular carcinoma: a review. J. Hepatocell. Carcinoma 3, 41-53 (2016)

15. Manzia, T. M. et al. Glycogen storage disease type la and VI associated with hepatocellular carcinoma: two case reports. Transplant. Proc. 43, 1181-1183 (2011).

16. Roscher, A. et al. The natural history of glycogen storage disease types VI and IX: long-term outcome from the largest metabolic center in Canada. Mol. Genet. Metab. 113, 171-176 (2014).

17. Albash, B. et al. Novel PHKG2 mutation causing GSD IX with prominent liver disease: report of three cases and review of literature. Eur. J. Pediatr. 173, 647-653 (2014).

18. Bali, D. S. et al. Variability of disease spectrum in children with liver phosphorylase kinase deficiency caused by mutations in the PHKG2 gene. Mol. Genet. Metab. 111, 309-313 (2014).

19. Zois, C. E., Favaro, E. \& Harris, A. L. Glycogen metabolism in cancer. Biochem. Pharmacol. 92, 3-11 (2014).

20. Yang, W. et al. Phosphorylase kinase $\beta$ represents a novel prognostic biomarker and inhibits malignant phenotypes of liver cancer cell. Int. J. Biol. Sci. 15, 2596-2606 (2019).

21. Zhou, D. et al. Clinical features and PHKG2 gene mutation analysis of 5 Chinese patients with glycogen storage disease IXc. J. Clin. Pediatr. 35, 609-612 (2017).

22. Waheed, N. et al. Variability of clinical and biochemical phenotype in liver phosphorylase kinase deficiency with variants in the phosphorylase kinase (PHKG2) gene. J. Pediatr. Endocrinol. Metab. 33, 1117-1123 (2020).

23. Beauchamp, N. J. et al. Glycogen storage disease type IX: high variability in clinical phenotype. Mol. Genet. Metab. 92, 88-99 (2007).

24. Michele, A. J. et al. Mutations in the testis/liver isoform of the phosphorylase kinase gamma subunit (PHKG2) cause autosomal liver glycogenosis in the GSD rat and in humans. Nat. Genet. 14, 337-340 (1996).

25. van Beurden, E. A. C. M. et al. Autosomal recessive liver phosphorylase kinase deficiency caused by a novel splice-site mutation in the gene encoding the liver gamma subunit (PHKG2). Biochem. Biophys. Res. Commun. 236, 544-548 (1997).

26. $\mathrm{Li}, \mathrm{C}$. et al. PHKG2 mutation spectrum in glycogen storage disease type IXc: a case report and review of the literature. J. Pediatr. Endocrinol. Metab. 31, 331-338 (2018).

27. Fahiminiya, S. et al. Whole exome sequencing unravels disease-causing genes in consanguineous families in Qatar. Clin. Genet. 86, 134-141 (2014).

\section{ACKNOWLEDGEMENTS}

We thank all staff in the Department of Pediatrics, Department of Gastroenterology and Hepatology, and Department of Gastroenterological Surgery, Kumamoto University Hospital for their help in clinical practice.

\section{AUTHOR CONTRIBUTIONS}

All contributors have read this manuscript and approved its submission for consideration, for publication as a Data Report in the Human Genome Variation. J. K. designed the case report and wrote the manuscript. J. K., H. M., T. W., and K. S. 
collected and analyzed data. J. K., H. M., and K. N. supervised this case report and clinical practice. H. S., and T. F. performed the PHKG2 gene analysis.

\section{FUNDING}

This work was supported in part by a Health and Labor Sciences Research Grant for Research on Rare and Intractable Diseases from the Ministry of Health, Labor and Welfare, Japan (grant number JPMH20FC1025); a Grant-in-Aid for Practical Research Project for Rare/Intractable Diseases from the Japan Agency for Medical Research and Development (AMED; grant numbers JP19ek0109276, JP21ek0109482); and a Grantin-Aid for Scientific Research from the Ministry of Education, Culture, Sports, Science, and Technology, Japan (Japan Society for the Promotion of Science [JSPS] KAKENHI: grant number JP20K08207). The funders had no role in the study design, data collection and analysis, decision to publish, or preparation of the manuscript.

\section{COMPETING INTERESTS}

The authors declare no competing interests.

\section{ADDITIONAL INFORMATION}

Supplementary information The online version contains supplementary material available at https://doi.org/10.1038/s41439-021-00172-8.
Correspondence and requests for materials should be addressed to Jun Kido.

Reprints and permission information is available at http://www.nature.com/ reprints

Publisher's note Springer Nature remains neutral with regard to jurisdictional claims in published maps and institutional affiliations.

cc) (i)

Open Access This article is licensed under a Creative Commons adaptation, distribution and reproduction in any medium or format, as long as you give appropriate credit to the original author(s) and the source, provide a link to the Creative Commons license, and indicate if changes were made. The images or other third party material in this article are included in the article's Creative Commons license, unless indicated otherwise in a credit line to the material. If material is not included in the article's Creative Commons license and your intended use is not permitted by statutory regulation or exceeds the permitted use, you will need to obtain permission directly from the copyright holder. To view a copy of this license, visit http://creativecommons. org/licenses/by/4.0/.

(c) The Author(s) 2021 